\title{
High mean platelet volume and neutrophil-to-lymphocyte ratio are predictors of mortality in patients with HIV-related non-Hodgkin's lymphoma
}

\author{
Juan Camilo Salgado Sánchez ${ }^{1}$, Jorge Leonardo Gamboa Quiñones ${ }^{1}$, \\ Oscar Mauricio Muñoz-Velandia ${ }^{1,2 *}$, Mónica Arévalo Zambrano ${ }^{2,3}$, \\ José Roberto Támara Ramírez $z^{1,2,4}$ \\ ${ }^{1}$ Department of Internal Medicine, Pontificia Universidad Javeriana, Bogotá, Colombia \\ ${ }^{2}$ Hospital Universitario San Ignacio, Bogotá, Colombia \\ ${ }^{3}$ Hematology Unit, Department of Internal Medicine, Pontificia Universidad Javeriana, Bogotá, Colombia \\ ${ }^{4}$ Infectology Unit, Department of Internal Medicine, Pontificia Universidad Javeriana, Bogotá, Colombia
}

\begin{abstract}
Introduction: Simple indices derived from a blood count are useful as predictors of survival in both solid and hematological tumors. However, these indices have not been studied in human immunodeficiency virus (HIV)-associated non-Hodgkin's lymphoma (NHL). The present study evaluates whether there are differences in the survival of such patients at two years according to baseline values of mean platelet volume (MPV) and neutrophil-to-lymphocyte ratio (NLR).
\end{abstract}

Material and methods: A retrospective cohort of patients between 2003 and 2018 with HIV and a confirmed diagnosis of some variant of nNHL at the Hospital Universitario San Ignacio, Bogotá, Colombia, was evaluated. 2-year survival was analyzed by comparing groups according to baseline values of MPV and NLR using Kaplan-Meier curves and log rank test. Cox regression model was used to control for confounding factors.

Results: 31 patients were included. The combined follow-up time was 602 months. Ten patients died during follow-up. The 2-year survival was lower for patients with MPV $>7.5 \mathrm{fL}$ (41.6 vs. $84.0 \%, p=0.01$, log rank test: 0.0057 ) and NLR $>4.35$ (33.0\% vs. $76.0 \%, p=0.04$; log rank test 0.028 ). Mortality was higher in patients with MPV >7.5 fL (HR 6.39; confidence interval $95 \% 1.35-30.21, p=0.015$ ) controlling by the effect of type of lymphoma, International Prognostic Index and functionality.

Conclusion: High values of MPV and NLR at the time of diagnosis of NHL are associated with mortality in patients with HIV infection. It is necessary to evaluate the utility of including these markers in prognostic indices.

Key words: non-Hodgkin's lymphoma, HIV, mean platelet volume, neutrophil-to-lymphocyte ratio

Acta Haematologica Polonica 2021; 52, 3: 195-201

\footnotetext{
*Address for correspondence: Oscar Muñoz-Velandia, Department of Internal Medicine, Hospital Universitario San Ignacio, Pontificia Universidad Javeriana, Cra. 7 \# 40-62. Office 713 Bogotá, Colombia, e-mail: o.munoz@javeriana.edu.co
} 


\section{Introduction}

Neoplasms such as non-Hodgkin's lymphoma (NHL) are frequent in patients with human immunodeficiency virus (HIV) infection. It is estimated that up to $40 \%$ of people with HIV develop a neoplasm during their lifetime [1] and about $33 \%$ of deaths in HIV patients are attributable to the presence of a neoplasm [2]. Non-Hodgkin's lymphoma is 60-200 times more common in HIV patients compared to the general population. Of these, $80 \%$ present as advanced systemic disease at the time of diagnosis [3]. However, the prognosis in these patients seems to be the same as in patients with or without HIV, achieving complete remission in $63.7 \%$ of patients with first line chemotherapy [4].

Several factors associated with increased mortality have been described in the population with NHL and HIV, such as: high viral load, low CD4 count at diagnosis, hypoalbuminemia $[5,6]$, coinfection with hepatotropic viruses [7], and the stage of the neoplasia defined with different scales such as the Ann Arbor classification [8] and the International Prognostic Index (IPI) [9].

In recent years, there has been growing interest in describing useful markers to predict adverse outcomes in patients with solid malignancies such as mean platelet volume (MPV) and neutrophil-to-lymphocyte ratio (NLR), given that increased values of NLR or low MPV are markers of tumor-related pro-inflammatory state, and are associated with a worse prognosis in patients with cancer of the endometrium [10], thyroid [11], breast [12], colon [13] and pancreas [14].

For HIV patients, an NLR $<2$ has been proposed as normal [15]. A relationship between mortality and increased NLR (>2) in patients with HIV seems clear, suggesting that worse prognosis is associated with the underlying systemic disturbance of the inflammatory process $[15,16]$. A meta-analysis that included 2,515 patients with diffuse large B-cell lymphoma showed that a high NLR prior to the start of treatment was associated with a higher mortality [hazard ratio (HR) 1.826; 95\% confidence interval (Cl): 1.238-2.692] [17].

A decreased platelet count and reduced MPV $(7.7 \mathrm{fL})$ has been reported for asymptomatic and treatment-naive HIV patients [18, 19], but behavior in patients with lymphoma has not been clearly described.

Specifically, MPV and NLR have not been studied in people who present simultaneously with HIV and NHL, despite there being simple, economical and widely available tests. The aim of the present study was to evaluate the survival of a cohort of patients with HIV and NHL according to the basal values of MPV and NLR in a reference center in Colombia.

\section{Material and methods}

A retrospective cohort study was conducted including patients over 18-years-old, managed in the HIV program of the Infectology Unit at the Hospital Universitario San
Ignacio, Bogotá, Colombia, between January 2003 and December 2018, with HIV infection confirmed according to the diagnostic algorithm proposed by the CDC [20], diagnosis of NHL confirmed with histopathology following the recommendations provided by the World Health Organization (WHO) for the diagnosis of hematolinfoid neoplasms [21], and with an available hemogram taken the time of diagnosis of NHL. Patients with autoimmune diseases, coronary heart disease, hyperthyroidism, or chronic kidney disease with GFR $<60 \mathrm{~mL} / \mathrm{min}$ were excluded, since these are conditions are associated with elevated MPV and NLR. This study was approved by the institutional ethics committee.

The information was collected and recorded systematically in the HIV program database. Patients managed in this program benefitted from a monthly follow-up with direct attention from a specialist in infectology, and six-monthly or even more frequent paraclinics if the presence of complications or any failure in therapy was detected. Blood count, taken at the time of lymphoma diagnosis, was processed in the same clinical laboratory for all patients using standardized techniques.

The NLR was calculated by dividing the absolute neutrophil count by the absolute lymphocyte count, and categorized as high or low using a cut-off threshold of 4.35 [22]. The MPV was obtained directly from the blood count, using Beckman Coulter DxH 600 equipment; a cut-off threshold of $7.5 \mathrm{fL}$ was used to define high MPV [23].

Mortality was assessed every 1-2 months depending on the patient's immunovirological status as stipulated by the institution's HIV program. To guarantee follow-up, a social worker from the Unit contacted patients if they did not attend the programmed controls.

We defined that death was attributable to lymphoma when patients did not reach full response according to the guidelines of the International Working Group [24]. Functionality was evaluated according to the classification suggested by the Eastern Cooperative Oncology Group (ECOG) [25]. The stage of disease was determined according to the IPI and the Ann Arbor classification [8].

The differences between the groups were evaluated with a $t$-test, a Mann-Whitney $U$ test or a chi-square test according to the nature of the variables. Survival functions were estimated according to the mentioned baseline MPV and NLR categories, using the Kaplan-Meier method. A nonparametric test (log-rank) was used to compare the survival functions of the different subgroups [26].

Finally, a multivariate Cox regression model was used to define whether MPV and NLR were associated with mortality after controlling for histological subtype, disease stage and baseline functionality. Analysis was performed with a Stata 14 statistical package (Stata Statistical Software: Release 14. College Station, TX: StataCorp LP). 
Table I. Demographic and clinical characteristics according to mean platelet volume (MPV)

\begin{tabular}{|c|c|c|c|}
\hline Variable & $\mathrm{MPV} \leq 7.5 \mathrm{fL}(19)$ & MPV >7.5 fL (12) & $p$ value \\
\hline Age, years (min-max) & $34(20-67)$ & $39(20-56)$ & 0.54 \\
\hline Hepatitis B, n [\%] & $3(15)$ & $2(16)$ & 0.94 \\
\hline \multicolumn{4}{|l|}{ Histology, n [\%]: } \\
\hline - DLBCL & $17(89.5)$ & $6(50)$ & \\
\hline - inmunoblastic & $0(0)$ & $2(16.6)$ & 0.04 \\
\hline - Burkitt & $2(10.5)$ & $2(16.6)$ & \\
\hline - plasmablastic & $0(0)$ & $2(16.6)$ & \\
\hline \multicolumn{4}{|l|}{ IPI, n [\%]: } \\
\hline - low & $3(16)$ & $2(16.6)$ & \\
\hline - intermediate low & $5(26)$ & $2(16.6)$ & 0.17 \\
\hline - intermediate high & $10(52)$ & $3(25)$ & \\
\hline - high & $1(5)$ & $5(41)$ & \\
\hline \multicolumn{4}{|l|}{ ECOG, n [\%] } \\
\hline - $0-2$ & $18(95)$ & $8(75)$ & 0.056 \\
\hline - $3-4$ & $1(5)$ & $4(25)$ & \\
\hline \multicolumn{4}{|c|}{ Staging (Ann Arbor), n [\%]: } \\
\hline$\cdot 1$ & $1(5)$ & $0(0)$ & \\
\hline - II & $2(10)$ & $0(0)$ & 0.25 \\
\hline - III & $3(15)$ & $1(8)$ & \\
\hline - IV & $13(67)$ & $11(92)$ & \\
\hline CD4* & 157,5 & 153 & 0.90 \\
\hline ART, n [\%]* & $14(63)$ & $8(66)$ & 0.675 \\
\hline Viral load (Log)* & $3.74(1.60-6.35)$ & $4.49(1.60-7.00)$ & 0.30 \\
\hline Albumin* & $3.35(2-4.4)$ & $3.32(2-4.7)$ & 0.83 \\
\hline
\end{tabular}

*The variables CD4; ART - viral load and albumin were taken at time of diagnosis of non-Hodgkin's lymphoma; fL - femtolitres; DLBCL - diffuse large B cell lymphoma; IPI - International Prognostic Index; ECOG - Eastern Cooperative Oncology Group; ART - antiretroviral therapy

\section{Results}

The baseline characteristics of the 31 patients according to the baseline MPV values are set out in Table I. Diffuse large B-cell lymphoma (DLBCL) was the most frequent histological subtype, representing almost $90 \%$ of cases with low MPV and $50 \%$ of cases with high MPV ( $p=0.04)$. A higher proportion of patients had impaired baseline functionality among patients with high MPV ( $25 \%$ vs. $5 \%, p=0.05)$.

Table Il shows the clinical and demographic characteristics of the patients according to the baseline NLR. $52 \%$ of patients with NLR $\leq 4.35$ were classified as high or high intermediate IPI, compared to $100 \%$ of patients with high NLR $(p=0.02)$.

The survival distribution function of patients with MPV $\leq 7.5 \mathrm{fL}$ was significantly higher (log rank test, $p=0.0057$ ) (Figure 1). The two-year survival of patients with MPV $>7.5$ was
$41.6 \%$ compared to $84 \%$ among patients with MPV $\leq 7.58$ $(p=0.01)$. Univariate analysis evaluating factors affecting mortality according to the Cox proportional hazard model showed that an MPV value $>7.5$ was associated with a greater risk (HR 5.43 95\% Cl: 1.39-21.15, $p=0.015$ ) (Table III), similar to that found in multivariate analysis after controlling by histological subtype, stage of disease and functionality (HR 6.39; 95\% Cl: 1.24-4.83, $p=0.015$ ) (Table IV).

Kaplan-Meier analysis of the survival distribution function according to the baseline NLR values is presented in Figure 2. A better survival function is evident among patients with NLR $\leq 4.35$ (log rank test, $p=0.028$ ). The 2 -year survival rate of patients with NLR $>4.35$ was $33 \%$ compared to $76 \%$ in those with low NLR $(p=0.04)$. Cox proportional hazard model showed that an NLR value $>4.35$ was 
Table II. Demographic and clinical characteristics according to neutrophil-to-lymphocyte ratio (NLR)

\begin{tabular}{|c|c|c|c|}
\hline Variable & NLR $\leq 4.35(25)$ & NLR $>4.35(6)$ & $p$ value \\
\hline Age, years, (min-max) & $34(20-56)$ & $49(25-67)$ & 0.08 \\
\hline Hepatitis B, n [\%] & $4(16)$ & $1(16)$ & 0.96 \\
\hline \multicolumn{4}{|l|}{ Histology, n [\%]: } \\
\hline - DLBCL & $19(76)$ & $4(66)$ & \\
\hline - inmunoblastic & $1(4)$ & $1(16.6)$ & 0.33 \\
\hline - Burkitt & $4(16)$ & $0(0)$ & \\
\hline - plasmablastic & $1(4)$ & $1(16.6)$ & \\
\hline IPI, n [\%]: & & & 0.02 \\
\hline - low & $5(20)$ & $2(0)$ & \\
\hline - intermediate low & $7(28)$ & $2(0)$ & \\
\hline - intermediate high & $11(44)$ & $2(33.3)$ & \\
\hline - high & $2(8)$ & $4(66.6)$ & \\
\hline ECOG, n [\%]: & & & 0.84 \\
\hline - $0-2$ & $21(84)$ & $6(100)$ & \\
\hline - $3-4$ & $4(16)$ & $0(0)$ & \\
\hline \multicolumn{4}{|c|}{ Staging (Ann Arbor), n [\%]: } \\
\hline$\cdot 1$ & $1(4)$ & $0(0)$ & \\
\hline • II & $2(8)$ & $0(0)$ & 0.97 \\
\hline$\cdot$ • III & $3(12)$ & $0(0)$ & \\
\hline$\cdot \mathrm{IV}$ & $18(72)$ & $6(100)$ & \\
\hline $\mathrm{CD} 4 *$ & 197.36 & 229 & 0.31 \\
\hline ART, n [\%]* & $16(64)$ & $6(100)$ & 0.08 \\
\hline Viral load (Log)* & $4.23(1.75-7.0)$ & $3.22(1.60-5.44)$ & 0.26 \\
\hline Albumin* & $3.38(2-4.7)$ & $3.15(2.6-4.2)$ & 0.36 \\
\hline
\end{tabular}

*The variables CD4; ART - viral load and albumin were taken at time of diagnosis of non-Hodgkin's lymphoma; fL - femtolitres; DLBCL - diffuse large B cell lymphoma; IPI - International Prognostic Index; ECOG - Eastern Cooperative Oncology Group; ART - antiretroviral therapy

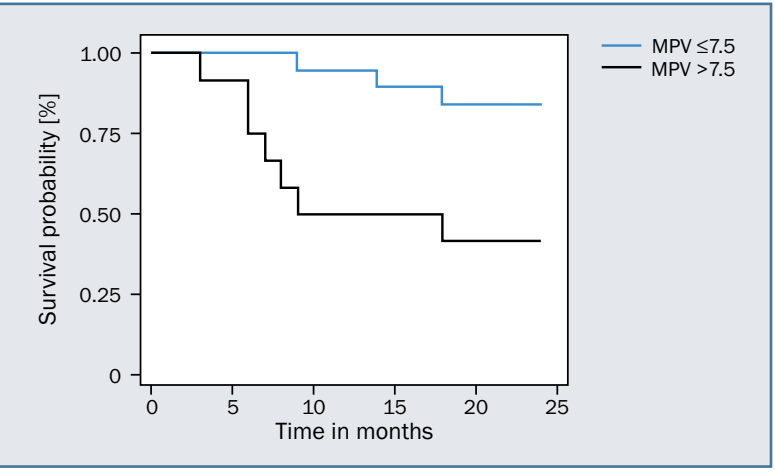

Figure 1. Survival (survivor) function estimated by Kaplan-Meier method, according to basal mean platelet volume; MPV - mean platelet volume

associated with higher mortality in univariate analysis (HR 3.73; 95\% Cl 1.04-13.36, $p=0.02$ ) (Table III), however this association was not significant in multivariate analysis (Table IV).
Table III. Factors affecting mortality according to Cox proportional hazard model. Univariate analysis at 24 months

\begin{tabular}{l|c|c}
\multirow{2}{*}{ Variable } & \multicolumn{2}{|c}{ Univariate analysis } \\
\cline { 2 - 3 } & HR (CI) & $p$ value \\
\hline High MPV & $5.43(1.39-21.15)$ & 0.015 \\
High NLR & $3.73(1.04-13.36)$ & 0.02 \\
Albumin & $1.32(0.42-4.34)$ & 0.68 \\
ECOG & $2.21(1.32-3.70)$ & 0.003 \\
Hepatitis B* & $1.52(0.32-7.16)$ & 0.59 \\
IPI & $4.30(1.77-10.45)$ & 0.001
\end{tabular}

*Hepatitis B infection; $\mathrm{HR}$ - hazard ratio; $\mathrm{Cl}$ - confidence interval; MPV - mean platelet volume; NLR - neutrophil-to-lymphocyte ratio; ECOG - Eastern Cooperative Oncology Group; IPI - International Prognostic Index

Additionally, multivariate analysis found a significant association between mortality and IPI (HR 2.87; 95\% Cl: 1.00-8.33, $p=0.05)$, and functionality evaluated using ECOG scale (HR 2.36; 95\% Cl: 1.20-4.64, $p=0.01$ ) 
Table IV. Factors affecting mortality according to Cox proportional hazard model. Multivariate mortality analysis at 24 months

\begin{tabular}{l|c|c|c|c|}
\hline \multirow{2}{*}{ Variable } & \multicolumn{2}{|c|}{ MPV } & \multicolumn{2}{c|}{ NLR } \\
\cline { 2 - 5 } & HR (Cl) & $p$ value & HR (CI) & $p$ value \\
\hline High & 6.39 & 0.015 & - & N/A \\
MPV & $(1.35-30.21)$ & & & \\
\hline High & - & N/A & 2.88 & 0.27 \\
NLR & & & $(0.43-19.17)$ & \\
\hline IPI & - & & 2.87 & 0.05 \\
\hline ECOG & 2.45 & $<0.01$ & $\begin{array}{c}(1.00-8.33) \\
2.36\end{array}$ & 0.01 \\
\hline
\end{tabular}

Controlling by clinical confounders; MPV - mean platelet volume; NLR - neutrophil-to-lymphocyte ratio; $\mathrm{HR}$ - hazard ratio; $\mathrm{Cl}$ - confidence interval; $\mathrm{N} / \mathrm{A}$ - not available; IPI - International Prognostic Index; ECOG - Eastern Cooperative Oncology Group

\section{Discussion}

This is the first study to evaluate MPV and NLR as prognostic markers in patients with NHL and HIV infection. We found that they are associated with survival at two years.

In recent years, a growing number of publications on inflammation biomarkers obtained from a blood count has appeared, with two being the most important described so far: MPV and NLR. These indices reflect the interaction that occurs between the tumor and the immune system.

The reason why lymphocyte is used within the denominator in the neutrophil-to-lymphocyte ratio is because this cell is the one that acts as an antitumor defense by suppressing tumor growth and dissemination, contrary to what happens with the neutrophil and platelet which have the ability to change their antitumor phenotype to a procarcinogenic and prometastatic phenotype [27-30].

The main mechanism used by the tumor cell to generate phenotypic change is cell-to-cell interaction through exosomes, sometimes called "friends of crime", which are vesicles that contain miRNA, RNA and proteins whose mission is to generate a change in the function of the target cell in order to promote an inflammatory environment from which the tumor cell will benefit [31].

When the tumor interacts with the neutrophil, it seeks to infiltrate healthy tissues both locally and remotely by providing enzymes that alter healthy connective tissue and express adhesion molecules that allow it to leave the intravascular compartment. Furthermore, by sharing exosomes with the tumor cell, it is capable of releasing said genetic material to the plasma by damaging the neutrophil extracellular traps (NET)osis process, generating more inflammation [27, 28].

A high MPV reflects the interaction between the tumor cell and the platelet, and its main role in cancer. When the platelet comes into contact with the tumor cell, it activates and changes its phenotype to a tumor-educated platelet

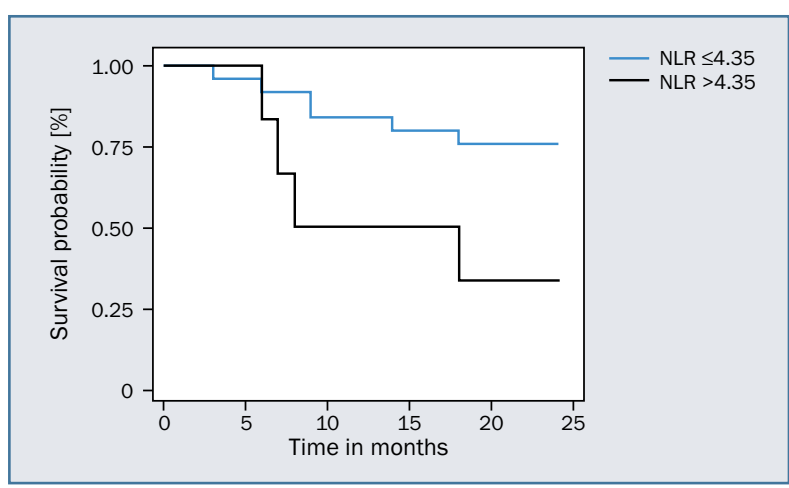

Figure 2. Survival (survivor) function estimated by Kaplan-Meier method, according to basal neutrophil-to-lymphocyte ratio (NLR)

(PET) [29], a cell with prometastatic function that acts as a 'trojan horse' by being a carrier of tumor genetic material which will generate distant sowings. It also shares proteins such as tissue factor and thrombin with the tumor cell that activate the coagulation cascade, and partly explains the pathophysiology of thromboembolic disease associated with cancer [29].

In addition, the activated platelet produces large quantities of reactive oxygen species (ROS), which are used by the tumor cell to generate hypoxia induced factor (HIF) and thereby block the mitochondria to prevent death by preventing the activation of caspases [32]. These two described biomarkers account at least partly for the complex interaction between the tumor cell and the blood cells.

Our study is the first to show that a high MPV is a predictor of poor outcomes in patients with NHL and HIV, even controlling for IPI and histological subtype of lymphoma. These results are similar to those reported by Rupa-Matysek in patients with Hodgkin's lymphoma, who found a trend to better survival in patients with low MPV $(<6.8 \mathrm{fL})$, although this finding did not reach statistical significance (log rank test $=1.124, p=0.2610$ ) [33]. However, our results are opposite to what Rupa-Matysek et al. reported in patients with diffuse large B-cell lymphoma and without HIV. According to them, a low MPV, defined as $\leq 9.1 \mathrm{fL}$, predicted higher mortality in patients with diffuse large B-cell lymphoma [17]. When low MPV was defined as $<10^{\text {th }}$ percentile (in that study $<6.1 \mathrm{fL}$ ), the HR for mortality was 5.56, $p<0.001$ [34]. A possible explanation is the inverse relationship between the MPV and the number of platelets, considering that a patient with HIV has thrombocytopenia as a manifestation of inflammation, unlike a person without infection where thrombocytosis would be observed. Furthermore, the decrease in baseline MPV values in HIV patients $[18,19]$ suggests that when MPV finally increases, it is associated with a severe inflammatory process. 
Similarly, our study is the first to evaluate the impact of baseline levels of NLR in patients with NHL and HIV. We found similar results to those reported by $\mathrm{Mu}$ et al. in patients without HIV. According to their meta-analysis, elevated NLR is an independent predictor of mortality with a HR of 1.826 (Cl: 1.238-2692) [17].

Our results suggest that MPV and NLR indices could have prognostic value to predict mortality in patients with HIV-associated NHL. However, the values seem to be different to those reported in other types of lymphoma. Further studies with a larger sample size and prospective cohorts are necessary to establish whether these indices could improve the prognostic ability of currently used scales like IPI or Ann Arbor.

The strengths of our study include the fact that the included patients completed the 2-year follow-up without losses to follow-up. There are also limitations, as the limited sample size decreases the precision of the effect estimate, although the published data on patients with HIV-associated NHL is very limited.

The cut-off thresholds used in our study allowed us to detect differences in mortality, but subsequent studies will be necessary to show whether MPV and NLR cut-off points have to be modified in order to improve predictive ability.

\section{Conclusion}

Biomarkers such as MPV and NLR are useful tools to define prognosis in patients with NHL associated with HIV, because they take into account the complex interaction that occurs between the tumor cell and blood cells.

\section{Authors' contributions}

JCS and JLG collected data and developed first draft of manuscript; MA provided independent review and confirmation of diagnoses; OMM assisted in analysis of data; OMM and JRT assisted in writing and editing of manuscript. All authors gave final approval and agreed to be accountable for this work.

\section{Conflict of interest}

The authors have no ethical conflicts to disclose.

\section{Financial support}

No external funding sources were needed.

\section{Ethics}

The work described in this article has been carried out in accordance with The Code of Ethics of the World Medical Association (Declaration of Helsinki) for experiments involving humans; EU Directive 2010/63/EU for animal experiments; Uniform Requirements for manuscripts submitted to Biomedical journals.

\section{References}

1. Akanmu AS. AIDS-associated malignancies. Afr J Med Med Sci. 2006; 35(Suppl): 57-70.

2. Vandenhende MA, Roussillon C, Henard S, et al. ANRS EN20 Mortalité 2010 study group. Cancer-related causes of death among HIV-Infected patients in France in 2010: evolution since 2000. PLoS One. 2015; 10(6): e0129550, doi: 10.1371/journal.pone.0129550, indexed in Pubmed: 26083524.

3. Grogg KL, Miller RF, Dogan A. HIV infection and lymphoma. J Clin Pathol. 2006; 60(12): 1365-1372, doi: 10.1136/jcp.2007.051953.

4. Schommers P, Gillor D, Hentrich M, et al. Incidence and risk factors for relapses in HIV-associated non-Hodgkin lymphoma as observed in the German HIV-related lymphoma cohort study. Haematologica. 2018; 103(5): 857-864, doi: 10.3324/haematol.2017.180893, indexed in Pubmed: 29439188.

5. Cuellar LE, Anampa-Guzmán A, Holguín AM, et al. Prognostic factors in HIV-positive patients with non-Hodgkin lymphoma: a Peruvian experience. Infect Agent Cancer. 2018; 13: 27, doi: 10.1186/s13027-0180200-y, indexed in Pubmed: 30083224.

6. Han X, Jemal A, Hulland E, et al. HIV infection and survival of lymphoma patients in the era of highly active antiretroviral therapy. Cancer Epidemiol Biomark Prev. 2016, doi: 10.1158/1055-9965.EPI-16-0595.

7. Marcucci F, Spada E, Mele A, et al. The association of hepatitis B virus infection with B-cell non-Hodgkin lymphoma - a review. Am J Blood Res. 2012; 2(1): 18-28, indexed in Pubmed: 22432084.

8. Armitage J0. Staging non-Hodgkin lymphoma. CA Cancer J Clin. 2005; 55(6): 368-376, doi: 10.3322/canjclin.55.6.368, indexed in Pubmed: 16282281.

9. Huang $\mathrm{HH}$, Xiao F, Chen FY, et al. Reassessment of the prognostic value of the International Prognostic Index and the revised International Prognostic Index in patients with diffuse large B-cell lymphoma: A multicentre study. Exp Ther Med. 2012; 4(3): 475-480, doi: 10.3892/ /etm.2012.607, indexed in Pubmed: 23181121.

10. Wang Y, Qiu H, Hu W, et al. Over-expression of platelet-derived growth factor-D promotes tumor growth and invasion in endometrial cancer. Int J Mol Sci. 2014; 15(3): 4780-4794, doi: 10.3390/ijms15034780, indexed in Pubmed: 24646915.

11. Baldane S, Ipekci SH, Sozen M, et al. Mean platelet volume could be a possible biomarker for papillary thyroid carcinomas. Asian Pac J Cancer Prev. 2015; 16(7): 2671-2674, doi: 10.7314/ /apjcp.2015.16.7.2671, indexed in Pubmed: 25854344.

12. Tanriverdi $\mathrm{O}$, Menekse S, Teker $\mathrm{F}$, et al. The mean platelet volume may predict the development of isolated bone metastases in patients with breast cancer: A retrospective study of the Young Researchers Committee of the Turkish Oncology Group (TOG). J BUON. 2016; 21(4): 840-850.

13. Tuncel T, Ozgun A, Emirzeoglu L, et al. Mean platelet volume as a prognostic marker in metastatic colorectal cancer patients treated with bevacizumab-combined chemotherapy. Asian Pac J Cancer Prev. 2014; 15(15): 6421-6423, doi: 10.7314/apjcp.2014.15.15.6421, indexed in Pubmed: 25124636.

14. Xue P, Kanai M, Mori Y, et al. Neutrophil-to-lymphocyte ratio for predicting palliative chemotherapy outcomes in advanced pancreatic cancer patients. Cancer Med. 2014; 3(2): 406-415, doi: 10.1002/ /cam4.204, indexed in Pubmed: 24519894.

15. Raffetti E, Donato F, Casari S, et al. Systemic inflammation-based scores and mortality for all causes in HIV-infected patients: a MASTER cohort study. BMC Infect Dis. 2017; 17(1): 193, doi: 10.1186/ /s12879-017-2280-5, indexed in Pubmed: 28264665. 
16. Hanberg JS, Freiberg MS, Goetz MB, et al. VACS Project Team. . Neutrophil-to-lymphocyte and platelet-to-lymphocyte ratios as prognostic inflammatory biomarkers in human immunodeficiency virus (HIV), hepatitis $\mathrm{C}$ virus (HCV), and HIV/HCV coinfection. Open Forum Infect Dis. 2019; 6(10): ofz347.

17. Mu S, Ai L, Fan F, et al. Prognostic role of neutrophil-to-lymphocyte ratio in diffuse large $B$ cell lymphoma patients: an updated dose-response meta-analysis. Cancer Cell Int. 2018; 18: 119, doi: 10.1186/ /s12935-018-0609-9, indexed in Pubmed: 30166942.

18. Nkambule BB, Davison GM, Ipp H. The evaluation of platelet indices and markers of inflammation, coagulation and disease progression in treatment-naïve, asymptomatic HIV-infected individuals. Int J Lab Hematol. 2015; 37(4): 450-458, doi: 10.1111/ijlh.12307, indexed in Pubmed: 25401263.

19. Qadri S, Holman S, Dehovitz J, et al. Mean platelet volume is decreased in HIV-infected women. HIV Med. 2013; 14(9): 549-555, doi: 10.1111/hiv.12048, indexed in Pubmed: 23738819.

20. Centers for Disease Control and Prevention and Association of Public Health Laboratories. Laboratory testing for the diagnosis of HIV infection: updated recommendations. http://stacks.cdc.gov/view/ /cdc/23447 (January 1, 2020).

21. Swerdlow SH, Campo E, Pileri SA, et al. The 2016 revision of the World Health Organization classification of lymphoid neoplasms. Blood. 2016; 127(20): 2375-2390, doi: 10.1182/blood-2016-01-643569, indexed in Pubmed: 26980727.

22. Templeton AJ, McNamara MG, Šeruga B, et al. Prognostic role of neutrophil-to-lymphocyte ratio in solid tumors: a systematic review and meta-analysis. J Natl Cancer Inst. 2014; 106(6): dju124, doi: 10.1093/jnci/dju124, indexed in Pubmed: 24875653.

23. Korniluk A, Koper-Lenkiewicz OM, Kamińska J, et al. Mean platelet volume (MPV): new perspectives for an old marker in the course and prognosis of inflammatory conditions. Mediators Inflamm. 2019; 2019: 9213074, doi: 10.1155/2019/9213074, indexed in Pubmed: 31148950.

24. Younes A, Hilden P, Coiffier B, et al. International Working Group consensus response evaluation criteria in lymphoma (RECIL 2017). Ann Oncol. 2017; 28(7): 1436-1447, doi: 10.1093/annonc/mdx097, indexed in Pubmed: 28379322.
25. Oken MM, Creech RH, Tormey DC, et al. Toxicity and response criteria of the Eastern Cooperative Oncology Group. Am J Clin Oncol. 1982; 5(6): 649-655, indexed in Pubmed: 7165009.

26. Hosmer DW, Lemeshow S, Sturdivant RX. Applied logistic regression. Third edition. John Wiley \& Sons, Inc., [City??] 2013.

27. Djordjevic D, Rondovic G, Surbatovic M, et al. Neutrophil-to-lymphocyte ratio, monocyte-to-lymphocyte ratio, platelet-to-lymphocyte ratio, and mean platelet volume-to-platelet count ratio as biomarkers in critically ill and injured patients: which ratio to choose to predict outcome and nature of bacteremia? Mediators Inflamm. 2018; 2018: 3758068 , doi: 10.1155/2018/3758068, indexed in Pubmed: 30116146.

28. Heeke S, Mograbi B, Alix-Panabières $C$, et al. Never travel alone: the crosstalk of circulating tumor cells and the blood microenvironment. Cells. 2019; 8(7), doi: 10.3390/cells8070714, indexed in Pubmed: 31337010 .

29. Palacios-Acedo AL, Mège D, Crescence L, et al. Platelets, thromboinflammation, and cancer: collaborating with the enemy. Front Immunol. 2019; 10: 1805, doi: 10.3389/fimmu.2019.01805, indexed in Pubmed: 31417569.

30. Xu XR, Yousef GM, Ni H. Cancer and platelet crosstalk: opportunities and challenges for aspirin and other antiplatelet agents. Blood. 2018; 131(16): 1777-1789, doi: 10.1182/blood-2017-05-743187, indexed in Pubmed: 29519806.

31. Apollonio B, Ramsay AG. Exosomes and CAFs: partners in crime. Blood. 2015; 126(9): 1053-1055, doi: 10.1182/blood-2015-07-655233, indexed in Pubmed: 26316614.

32. Qiu GZ, Jin MZ, Dai JX, et al. Reprogramming of the tumor in the hypoxic niche: the emerging concept and associated therapeutic strategies. Trends Pharmacol Sci. 2017; 38(8): 669-686, doi: 10.1016/j. tips.2017.05.002, indexed in Pubmed: 28602395.

33. Rupa-Matysek J, Gil L, Barańska M, et al. Mean platelet volume as a predictive marker for venous thromboembolism in patients treated for Hodgkin lymphoma. Oncotarget. 2018; 9(30): 21190-21200, doi: 10.18632/oncotarget.25002, indexed in Pubmed: 29765530.

34. Rupa-Matysek J, Gil L, Kroll-Balcerzak R, et al. Mean platelet volume as a predictive marker for venous thromboembolism and mortality in patients treated for diffuse large B-cell lymphoma. Hematol Oncol. 2017; 35(4): 456-464, doi: 10.1002/hon.2321, indexed in Pubmed: 27377517. 